Research Article

\title{
Bounds on the $\alpha$-Distance Energy and $\alpha$-Distance Estrada Index of Graphs
}

\author{
Yang Yang, ${ }^{1}$ Lizhu Sun, ${ }^{2}$ and Changjiang Bu $\mathbb{D}^{1,2}$ \\ ${ }^{1}$ College of Automation, Harbin Engineering University, Harbin 150001, PR, China \\ ${ }^{2}$ College of Mathematical Sciences, Harbin Engineering University, Harbin 150001, PR, China
}

Correspondence should be addressed to Changjiang Bu; buchangjiang@hrbeu.edu.cn

Received 31 October 2019; Revised 27 January 2020; Accepted 3 February 2020; Published 29 April 2020

Academic Editor: Manuel De la Sen

Copyright (c) 2020 Yang Yang et al. This is an open access article distributed under the Creative Commons Attribution License, which permits unrestricted use, distribution, and reproduction in any medium, provided the original work is properly cited.

Let $G$ be a simple undirected connected graph, then $D_{\alpha}(G)=\alpha \operatorname{Tr}(G)+(1-\alpha) D(G)$ is called the $\alpha$-distance matrix of $G$, where $\alpha \in[0,1], D(G)$ is the distance matrix of $G$, and $\operatorname{Tr}(G)$ is the vertex transmission diagonal matrix of $G$. In this paper, we study some bounds on the $\alpha$-distance energy and $\alpha$-distance Estrada index of $G$. Furthermore, we establish the relation between $\alpha$-distance Estrada index and $\alpha$-distance energy.

\section{Introduction}

1.1. $\alpha$-Distance Energy of Graphs. In this paper, we suppose that $G$ is a connected graph. Let $G=(V(G), E(G))$ be a graph with the vertex set $V(G)=\left\{v_{1}, \ldots, v_{n}\right\}$ and edge set $E(G)$. The distance between two vertices $v_{i}, v_{j} \in V(G)$ is the length of the shortest path between $v_{i}$ and $v_{j}$, denoted by $d\left(v_{i}, v_{j}\right)$. The Wiener index $W(G)$ of the graph $G$ is $W(G)=(1 / 2) \sum_{v_{i}, v_{j} \in V(G)} d\left(v_{i}, v_{j}\right)$. The matrix $D(G)=$ $\left(d_{i, j}\right) \in \mathscr{R}^{n \times n}$ is called the distance matrix of $G$, where $d_{i, j}=d\left(v_{i}, v_{j}\right), i, j \in\{1,2, \ldots, n\}$. For some properties of distance matrix, see [1-3].

The adjacency matrix of the graph $G$ is $\widetilde{A}(G)=\left(\widetilde{a}_{i j}\right) \in \mathscr{R}^{n \times n}$, where $\widetilde{a}_{i j}=1$ if $(i, j) \in E(G)$ and $\widetilde{a}_{i j}=$ 0 otherwise. The Laplacian matrix and signless Laplacian matrix of $G$ are $\widetilde{L}(G)=\widetilde{D}(G)-\widetilde{A}(G)$ and $\widetilde{Q}(G)=$ $\widetilde{D}(G)+\widetilde{A}(G)$, respectively, where $\widetilde{D}(G)=\operatorname{diag}\left(\widetilde{d}_{v_{1}}, \ldots\right.$, $\left.\widetilde{d}_{v_{n}}\right) \in \mathscr{R}^{n \times n}$ and $\widetilde{d}_{v_{i}}$ is the degree of $v_{i}, i=1,2, \ldots, n$.

In 2013, the study of Laplacian matrix and signless Laplacian matrix was extended to distance Laplacian matrices and distance signless Laplacian matrices defined as in equation (1) (see [4]). In 2016, the study of the spectrum of signless Laplacian matrix was generalized to a convex combination of $\widetilde{D}(G)$ and $\widetilde{A}(G)$ defined as $A_{(\alpha)}(G)=\alpha \widetilde{D}(G)+(1-\alpha) \widetilde{A}(G), \alpha \in[0,1]$ (see [5]). In [6], the above study was further extended to the $\alpha$-distance matrices (see equation (2)).

Let $\operatorname{Tr}\left(v_{i}\right)=\sum_{v_{j} \in V(G)} d\left(v_{i}, v_{j}\right)$ is called the transmission of $v_{i}$. Let

$$
\begin{aligned}
\mathscr{L}(G) & =\operatorname{Tr}(G)-D(G), \\
\mathscr{Q}(G) & =\operatorname{Tr}(G)+D(G),
\end{aligned}
$$

where $\operatorname{Tr}(G)=\operatorname{diag}\left(\operatorname{Tr}\left(v_{1}\right), \ldots, \operatorname{Tr}\left(v_{n}\right)\right) . \mathscr{L}(G)$ and $\mathscr{Q}$ are called the distance Laplacian matrix and distance signless Laplacian matrix of the graph $G$, respectively. A graph $G$ is said to be transmission regular if the transmissions of all the vertices in $V(G)$ are equal (see [4]). For a transmission regular graph $G$, the characteristic polynomials of $\mathscr{L}(G)$ and Q $(G)$ were characterized in [4]. For more properties of $\mathscr{L}(G)$ and $\mathscr{Q}(G)$, see [7-9].

In [6], the $\alpha$-distance matrix of a graph $G$

$$
D_{(\alpha)}(G)=\alpha \operatorname{Tr}(G)+(1-\alpha) D(G), \quad \alpha \in[0,1],
$$

was defined. Clearly, $\quad D_{(0)}(G)=D(G), \quad D_{(1 / 2)}(G)=$ $(1 / 2) \mathscr{Q}(G), \quad D_{(1)}(G)=\operatorname{Tr}(G)$, and $D_{(\alpha)}(G)-D_{(\beta)}(G)=$ $(\alpha-\beta) \mathscr{L}(G)$. The spectra of $D_{(\alpha)}(G)$ is called the $\alpha$-distance spectra of $G$. Since $D_{(\alpha)}(G)$ is a real symmetric matrix, the eigenvalues of $D_{(\alpha)}(G)$ are real. Let $\sigma_{(1)}^{\alpha}(G) \geq \sigma_{(2)}^{\alpha}$ $(G) \geq \cdots \geq \sigma_{(n)}^{\alpha}(G)$ be the eigenvalues of $D_{(\alpha)}(G)$. And let $\rho_{(\alpha)}(G)$ denote the spectral radius of $D_{(\alpha)}(G)$. From the 
Perron-Frobenius theorem, we have $\sigma_{(1)}(G)=\rho_{\alpha}(G)$. The spectral properties of $D_{(\alpha)}(G)$ were recently studied including spectral radius, second largest eigenvalue, $k$-th smallest eigenvalue, and smallest eigenvalue (see $[6,10-12]$ ).

Graph energy is an important graph invariant in graph theory; some graph energies $E_{\pi}(G)=\sum_{i=1}^{n}\left|\lambda_{i}(G)\right|, D E(G)$ $=\sum_{i=1}^{n}\left|\gamma_{i}(G)\right|$, and DSLE $(G)=\sum_{i=1}^{n}\left|\nu_{i}(G)-(2 W(G) / n)\right|$ are called the energy (original energy), the distance energy, and the distance signless Laplacian energy, respectively, where $\lambda_{i}(G), \gamma_{i}(G)$, and $\nu_{i}(G)$ denote the eigenvalues of $\widetilde{A}(G)$, $D(G)$, and $Q(G)$, respectively, $i=1,2, \ldots, n$ and $n=|V(G)|$ (see [13-16]).

Graph energy has important applications in the fields of mathematics and chemistry. There are many research studies on the above kinds of graph energy. Scholars gave the bounds on the energy of graphs, for example, the McClelland's bounds [17], Koolen-Moulton's bounds [18] and so on [19]. In [16], the distance energy of some graphs was calculated.

In [11], Guo and Zhou extended the concept of graph energy to a more general form called $\alpha$-distance energy:

$$
\varsigma_{(\alpha)}(G)=\sum_{i=1}^{n}\left|\sigma_{(i)}^{\alpha}(G)-\frac{2 \alpha W(G)}{n}\right|, \quad \alpha \in[0,1],
$$

where $\sigma_{(i)}^{\alpha}(G)$ is the eigenvalue of $D_{(\alpha)}(G), i=1,2, \ldots, n$, $n=|V(G)|$. Clearly, $\quad \varsigma_{(0)}(G)=D E(G)$ and $\varsigma_{(1 / 2)}(G)=$ $(1 / 2) \operatorname{DSLE}(G)$.

1.2. $\alpha$-Distance Estrada Index of Graphs. In [20], a spectral quantity is put forward by Estrada. $\operatorname{EE}(G)=\sum_{i=1}^{n} e^{\lambda_{i}(G)}=$ $\sum_{i=1}^{n} \sum_{k=0}^{\infty} \lambda_{i}^{k}(G) / k$ ! is called the Estrada index of $G$, where $\lambda_{1}(G), \lambda_{2}(G), \ldots, \lambda_{n}(G)$ denote the eigenvalues of $\widetilde{A}(G)$ (see [20]). It is well-known that the Estrada index plays an important role in the problem of characterizing the molecular structure [21] and complex networks [22-25]. In [26], the study was extended to distance matrices, and the distance Estrada index of $G$ is $\operatorname{DEE}(G)=$ $\sum_{i=1}^{n} e^{\gamma_{i}(G)}=\sum_{i=1}^{n} \sum_{k=0}^{\infty} \gamma_{i}^{k}(G) / k !$ !, where $\gamma_{1}(G), \gamma_{2}(G), \ldots$, $\gamma_{n}(G)$ are the eigenvalues of $D(G)$. Let

In this paper, we consider a more general Estrada index.

$$
\operatorname{DEE}_{(\alpha)}(G)=\sum_{i=1}^{n} e^{\sigma_{(i)}^{\alpha}(G)}=\sum_{i=1}^{n} \sum_{k=0}^{\infty} \frac{\left(\sigma_{(i)}^{\alpha}(G)\right)^{k}}{k !},
$$

be the $\alpha$-distance Estrada index of $G$, where $\sigma_{(1)}^{\alpha}(G), \ldots, \sigma_{(n)}^{\alpha}(G)$ are the eigenvalues of $D_{(\alpha)}(G)$. Clearly, $\operatorname{DEE}_{(0)}(G)=\operatorname{DEE}(G)$.

1.3. Main Work. In this paper, we study some bounds on the $\alpha$-distance energy and $\alpha$-distance Estrada index of graphs in terms of the parameter $\alpha$ and the vertex number, the transmission of vertices and Wiener index. Furthermore, we establish the relation between $\alpha$-distance Estrada index and $\alpha$-distance energy.

\section{Some Bounds for the $\alpha$-Distance Energy of Graphs}

To begin with this section, we introduce some notations and propositions.

Proposition 1 (see [6]). Let $G$ be a graph with $n$ vertices. Then,

$$
\begin{gathered}
\sum_{i=1}^{n} \sigma_{(i)}^{\alpha}(G)=\sum_{i=1}^{n} \alpha \operatorname{Tr}\left(v_{i}\right)=2 \alpha W(G), \\
\sum_{i=1}^{n}\left(\sigma_{(i)}^{\alpha}(G)\right)^{2}=\alpha^{2} \sum_{i=1}^{n} \operatorname{Tr}^{2}\left(v_{i}\right)+2(1-\alpha)^{2} S
\end{gathered}
$$

where $S=\sum_{1 \leq i<j \leq n} d^{2}\left(v_{i}, v_{j}\right)$ and $\sigma_{(1)}^{\alpha}(G) \geq \sigma_{(2)}^{\alpha}(G) \geq \cdots \geq$ $\sigma_{(n)}^{\alpha}(G)$ denotes the eigenvalues of $D_{(\alpha)}(G)$.

In the following, a new matrix is established:

$$
U_{(\alpha)}(G)=\alpha \operatorname{Tr}(G)+(1-\alpha) D(G)-\frac{2 \alpha W(G)}{n} I_{n}, \quad \alpha \in[0,1],
$$

where $I_{n}$ denotes identity matrix of order $n$. Let $\eta_{(1)}^{\alpha}(G), \eta_{(2)}^{\alpha}(G), \ldots, \eta_{(n)}^{\alpha}(G)$ denote the eigenvalues of $U_{(\alpha)}(G)$. Obviously,

$$
\varsigma_{(\alpha)}(G)=\sum_{i=1}^{n}\left|\eta_{(i)}^{\alpha}(G)\right|, \quad \alpha \in[0,1] .
$$

Proposition 2. Let $G$ be a graph with $n$ vertices. Then,

$$
\begin{aligned}
& \sum_{i=1}^{n} \eta_{(i)}^{\alpha}(G)=0, \sum_{i=1}^{n}\left(\eta_{(i)}^{\alpha}(G)\right)^{2}=2 Z \\
& Z=-\sum_{1 \leq i<j \leq n} \eta_{(i)}^{\alpha}(G) \eta_{(j)}^{\alpha}(G)=\left|\sum_{1 \leq i<j \leq n} \eta_{(i)}^{\alpha}(G) \eta_{(j)}^{\alpha}(G)\right|
\end{aligned}
$$

where $Z=(1-\alpha)^{2} S+\left(\alpha^{2} / 2\right) \sum_{i=1}^{n}\left(\operatorname{Tr}\left(v_{i}\right)-(2 W(G) / n)\right)^{2}$ and $\eta_{(1)}^{\alpha}(G), \eta_{(2)}^{\alpha}(G), \ldots, \eta_{(n)}^{\alpha}(G)$ denote the eigenvalues of $U_{(\alpha)}(G)$.

Proof. In order to prove equation (8), let $\eta_{(1)}^{\alpha}(G), \eta_{(2)}^{\alpha}(G), \ldots, \eta_{(n)}^{\alpha}(G)$ denote the eigenvalues of $U_{(\alpha)}(G)$, by equations (5) and (6), we have

$$
\begin{aligned}
\sum_{i=1}^{n}\left(\eta_{(i)}^{\alpha}(G)\right)^{2}= & \operatorname{trace}\left(U_{(\alpha)}^{2}(G)\right)=2(1-\alpha)^{2} S \\
& +\alpha^{2} \sum_{i=1}^{n}\left(\operatorname{Tr}\left(v_{i}\right)-\frac{2 W(G)}{n}\right)^{2} .
\end{aligned}
$$

By equation (5), we have

$$
0=\left(\sum_{i=1}^{n} \eta_{(i)}^{\alpha}(G)\right)^{2}=2 Z+2 \sum_{1 \leq i<j \leq n} \eta_{(i)}^{\alpha}(G) \eta_{(j)}^{\alpha}(G) .
$$

Then, 


$$
Z=-\sum_{1 \leq i<j \leq n} \eta_{(i)}^{\alpha}(G) \eta_{(j)}^{\alpha}(G)=\left|\sum_{1 \leq i<j \leq n} \eta_{(i)}^{\alpha}(G) \eta_{(j)}^{\alpha}(G)\right| .
$$

In the following, we introduce some Lemmas which are helpful for the following proofs of theorems.

Lemma 1 (see [6]). Let $G$ be a graph with $n$ vertices. Then,

$$
\rho_{\alpha}(G) \geq \frac{2 W(G)}{n},
$$

the equality holds if and only if $G$ is a transmission regular graph.

Lemma 2 (see [27]). Let $G$ be a graph with $n$ vertices. Then,

$$
W(G) \geq \frac{n(n-1)}{2},
$$

the equality holds if and only if $G \cong K_{n}$. $K_{n}$ denotes a complete graph with $n$ vertices.

Next, we give some bounds for the $\alpha$-distance energy of a graph by using the parameter $\alpha$ and the vertex number.

Theorem 1. Let $G$ be a connected graph with $n$ vertices. Then,

$$
\varsigma_{\alpha}(G) \geq 2(1-\alpha)(n-1),
$$

the equality holds if and only if $G \cong K_{n}$.

Proof. Let $\sigma_{(1)}^{\alpha}(G) \geq \sigma_{(2)}^{\alpha}(G) \geq \ldots \geq \sigma_{(n)}^{\alpha}(G)$ be the eigenvalues of $D_{\alpha}(G)$.

By Lemma 1 and $\alpha \in[0,1]$, we know that $\sigma_{(1)}^{\alpha}(G) \geq(2 W(G) / n) \geq(2 W(G) \alpha / n)$. Suppose that $\iota$ is the largest number such that $\sigma_{(l)}^{\alpha}(G) \geq(2 W(G) \alpha / n)$. It follows from equation (5) that

$$
\begin{aligned}
& \varsigma_{(\alpha)}(G)= \sum_{i=1}^{\iota}\left(\sigma_{(i)}^{\alpha}(G)-\frac{2 W(G) \alpha}{n}\right)+\sum_{i=\iota+1}^{n}\left(\frac{2 W(G) \alpha}{n}-\sigma_{(i)}^{\alpha}(G)\right) \\
&= \sum_{i=1}^{\iota} \sigma_{(i)}^{\alpha}(G)-\imath \frac{2 W(G) \alpha}{n}+(n-\imath) \frac{2 W(G) \alpha}{n}-\sum_{i=l+1}^{n} \sigma_{(i)}^{\alpha}(G) \\
&= \sum_{i=1}^{\iota} \sigma_{(i)}^{\alpha}(G)-\imath \frac{2 W(G) \alpha}{n}-\imath \frac{2 W(G) \alpha}{n}+\sum_{i=1}^{n} \sigma_{(i)}^{\alpha}(G) \\
&-\sum_{i=l+1}^{n} \sigma_{(i)}^{\alpha}(G) \\
&= 2 \sum_{i=1}^{l}\left(\sigma_{(i)}^{\alpha}(G)-\frac{2 W(G) \alpha}{n}\right) \\
& \geq 2\left(\sigma_{(1)}^{\alpha}(G)-\frac{2 W(G) \alpha}{n}\right) .
\end{aligned}
$$

From Lemmas 1 and 2, we have

$$
\begin{aligned}
& 2\left(\sigma_{(1)}^{\alpha}(G)-\frac{2 W(G) \alpha}{n}\right) \geq 2\left(\frac{2 W(G)}{n}-\alpha \frac{2 W(G)}{n}\right) \\
& \quad=4(1-\alpha) \frac{W(G)}{n} \geq 2(1-\alpha)(n-1) .
\end{aligned}
$$

The above three inequalities are the equality holds if and only if $G \cong K_{n}$.

We give some bounds for $\alpha$-distance energy through the order $n$, the transmission of vertex and the parameter $\alpha$ based on Cauchy-Schwarz inequalities in the following.

Theorem 2. Let $G$ be a graph with $n$ vertices. Then,

$$
\sqrt{2 Z+n(n-1) p^{(2 / n)}} \leq \varsigma_{(\alpha)}(G) \leq \sqrt{2 n Z},
$$

where $p=\operatorname{det}\left|U_{(\alpha)}(G)\right|$ and $Z=(1-\alpha)^{2} S+\left(\alpha^{2} / 2\right) \sum_{i=1}^{n}$ $\left(\operatorname{Tr}\left(v_{i}\right)-(2 W(G) / n)\right)^{2}$.

Proof. Let $\eta_{(1)}^{\alpha}(G), \eta_{(2)}^{\alpha}(G), \ldots, \eta_{(n)}^{\alpha}(G)$ denote the eigenvalues of $U_{(\alpha)}(G)$. From Cauchy-Schwarz inequality, we have

$$
\left(\varsigma_{(\alpha)}(G)\right)^{2}=\left(\sum_{i=1}^{n}\left|\eta_{(i)}^{\alpha}(G)\right| \cdot 1\right)^{2} \leq \sum_{i=1}^{n}\left|\eta_{(i)}^{\alpha}(G)\right|^{2} \sum_{i=1}^{n} 1 .
$$

Using equations (7) and (8), we have

$$
\left(\varsigma_{(\alpha)}(G)\right)^{2} \leq 2 n Z \text {. }
$$

So,

$$
\varsigma_{(\alpha)}(G) \leq \sqrt{2 n Z}
$$

Similarly, from equation (11), we know

$$
\begin{aligned}
\left(\varsigma_{(\alpha)}(G)\right)^{2}= & \left(\sum_{i=1}^{n}\left|\eta_{(i)}^{\alpha}(G)\right|\right)^{2}=\sum_{i=1}^{n}\left|\eta_{(i)}^{\alpha}(G)\right|^{2} \\
& +\sum_{i \neq j}\left|\eta_{(i)}^{\alpha}(G)\right|\left|\eta_{(j)}^{\alpha}(G)\right| .
\end{aligned}
$$

According to arithmetic-geometric inequality, we have

$$
\begin{gathered}
\frac{1}{n(n-1)} \sum_{i \neq j}\left|\eta_{(i)}^{\alpha}(G) \| \eta_{(j)}^{\alpha}(G)\right| \geq \prod_{i \neq j}\left(\left|\eta_{(i)}^{\alpha}(G) \| \eta_{(j)}^{\alpha}(G)\right|\right)^{(1 / n(n-1))} \\
=\left(\prod_{i=1}^{n}\left|\eta_{(i)}^{\alpha}(G)\right|^{(n-1)} \prod_{j=1}^{n}\left|\eta_{(j)}^{\alpha}(G)\right|^{(n-1)}\right)^{(1 / n(n-1))} \\
=\left(\prod_{i=1}^{n}\left|\eta_{(i)}^{\alpha}(G)\right|^{2(n-1)}\right)^{(1 / n(n-1))}=\left|\prod_{i=1}^{n} \eta_{(i)}^{\alpha}(G)\right|^{(2 / n)} .
\end{gathered}
$$

By equations (7) and (9), we have

$$
\begin{aligned}
\left(\varsigma_{(\alpha)}(G)\right)^{2}=\sum_{i=1}^{n}\left|\eta_{(i)}^{\alpha}(G)\right|^{2} & +\sum_{i \neq j}\left|\eta_{(i)}^{\alpha}(G)\right|\left|\eta_{(j)}^{\alpha}(G)\right| \\
& \geq 2 Z+n(n-1) p^{(2 / n)}
\end{aligned}
$$


where $p=\operatorname{det}\left|U_{(\alpha)}(G)\right|$.

So,

$$
\sqrt{2 Z+n(n-1) p^{(2 / n)}} \leq \varsigma_{(\alpha)}(G) \leq \sqrt{2 n Z} .
$$

In the following, we can obtain another lower bound in terms of the vertex number and the maximum value of $\left|\eta_{(i)}^{\alpha}(G)\right|$ of $U_{(\alpha)}(G)$.

Corollary 1. Let $G$ be a graph with $n$ vertices, then

$$
\varsigma_{(\alpha)}(G) \leq \delta_{(1)}^{\alpha}(G)+\sqrt{(n-1)\left(2 Z-\delta_{(1)}^{\alpha}(G)^{2}\right)},
$$

where $\delta_{(1)}^{\alpha}(G)=\max \left\{\left|\eta_{(i)}^{\alpha}(G)\right|\right\} \quad$ and $\quad Z=(1-\alpha)^{2} S+$ $\left(\alpha^{2} / 2\right) \sum_{i=1}^{n}\left(\operatorname{Tr}\left(v_{i}\right)-(2 W(G) / n)\right)^{2}$.

Proof. Let $\delta_{(1)}^{\alpha}(G) \geq \delta_{(2)}^{\alpha}(G) \geq, \ldots, \geq \delta_{(n)}^{\alpha}(G)$ be a nonincreasing sequence of $\left|\eta_{(i)}^{\alpha}(G)\right|$. From Cauchy-Schwarz inequality, we have

$$
\left(\sum_{i=2}^{n} \delta_{(i)}^{\alpha}(G) \cdot 1\right)^{2} \leq \sum_{i=2}^{n}\left(\delta_{(i)}^{\alpha}(G)\right)^{2} \sum_{i=2}^{n} 1 .
$$

By equation (7), we have

$$
\left(\varsigma_{(\alpha)}(G)-\delta_{(1)}^{\alpha}(G)\right)^{2} \leq(n-1)\left(2 Z-\left(\delta_{(1)}^{\alpha}(G)\right)^{2}\right) .
$$

So,

$$
\varsigma_{(\alpha)}(G) \leq \delta_{(1)}^{\alpha}(G)+\sqrt{(n-1)\left(2 Z-\left(\delta_{(1)}^{\alpha}(G)\right)^{2}\right)} .
$$

In the following, we obtained some new bounds for $\alpha$-distance energy through the Ozeki [28] and Polya's [29] inequality, respectively.

Lemma 3 (see [29]). Suppose $a_{i}$ and $b_{i}$ are real numbers for $1 \leq i \leq n$, then

$$
\left(\sum_{i=1}^{n} a_{i}^{2}\right)\left(\sum_{i=1}^{n} b_{i}^{2}\right) \leq \frac{1}{4}\left(\sqrt{\frac{M_{1} M_{2}}{m_{1} m_{2}}}+\sqrt{\frac{m_{1} m_{2}}{M_{1} M_{2}}}\right)^{2}\left(\sum_{i=1}^{n} a_{i} b_{i}\right)^{2},
$$

where $M_{1}=\max _{1 \leq i \leq n} a_{i}, M_{2}=\max _{1 \leq i \leq n} b_{i}, m_{1}=\min _{1 \leq i \leq n} a_{i}$, and $m_{2}=\min _{1 \leq i \leq n} b_{i}$.

Lemma 4 (see [28]). If $a_{i}$ and $b_{i}$ are real numbers for $1 \leq i \leq n$, then

$$
\left(\sum_{i=1}^{n} a_{i}^{2}\right)\left(\sum_{i=1}^{n} b_{i}^{2}\right)-\left(\sum_{i=1}^{n} a_{i} b_{i}\right)^{2} \leq \frac{n^{2}}{4}\left(M_{1} M_{2}-m_{1} m_{2}\right)^{2},
$$

where $M_{1}=\max _{1 \leq i \leq n} a_{i}, M_{2}=\max _{1 \leq i \leq n} b_{i}, m_{1}=\min _{1 \leq i \leq n} a_{i}$, and $m_{2}=\min _{1 \leq i \leq n} b_{i}$.

Theorem 3. Let $G$ be a graph with $n$ vertices. Then,

$$
\varsigma_{(\alpha)}(G) \geq \frac{2 \sqrt{2 n Z} \sqrt{\delta_{(1)}^{\alpha}(G) \delta_{(n)}^{\alpha}(G)}}{\delta_{(1)}^{\alpha}(G)+\delta_{(n)}^{\alpha}(G)}
$$

where $\delta_{(1)}^{\alpha}(G)$ and $\delta_{(n)}^{\alpha}(G)$ are the largest and the smallest of $\left|\eta_{(i)}^{\alpha}(G)\right|, \quad$ respectively, and $Z=(1-\alpha)^{2} S+\left(\alpha^{2} / 2\right)$ $\sum_{i=1}^{n}\left(\operatorname{Tr}\left(v_{i}\right)-(2 W(G) / n)\right)^{2}$.

Proof. Let $\delta_{(1)}^{\alpha}(G) \geq \delta_{(2)}^{\alpha}(G) \geq, \ldots, \geq \delta_{(n)}^{\alpha}(G)$ be a nonincreasing sequence of $\left|\eta_{(i)}^{\alpha}(G)\right|$ and let $a_{i}=\delta_{(i)}^{\alpha}(G)$ and $b_{i}=1$, where $i=1, \ldots, n$. By Lemma 3 , we have

$$
\begin{aligned}
\left(\sum_{i=1}^{n}\left(\delta_{(i)}^{\alpha}(G)\right)^{2}\right)\left(\sum_{i=1}^{n} 1\right) \leq & \frac{1}{4}\left(\sqrt{\frac{\delta_{(1)}^{\alpha}(G)}{\delta_{(n)}^{\alpha}(G)}}+\sqrt{\frac{\delta_{(n)}^{\alpha}(G)}{\delta_{(1)}^{\alpha}(G)}}\right)^{2} \\
& \cdot\left(\sum_{i=1}^{n} \delta_{(i)}^{\alpha}(G)\right)^{2} .
\end{aligned}
$$

By equation (8), we have

$$
2 n Z \leq \frac{1}{4}\left(\sqrt{\frac{\delta_{(1)}^{\alpha}(G)}{\delta_{(n)}^{\alpha}(G)}}+\sqrt{\frac{\delta_{(n)}^{\alpha}(G)}{\delta_{(1)}^{\alpha}(G)}}\right)^{2}\left(\varsigma_{(\alpha)}(G)\right)^{2} .
$$

Thus,

$$
\varsigma_{(\alpha)}(G) \geq \frac{2 \sqrt{2 n Z} \sqrt{\delta_{(1)}^{\alpha}(G) \delta_{(n)}^{\alpha}(G)}}{\delta_{(1)}^{\alpha}(G)+\delta_{(n)}^{\alpha}(G)} .
$$

Theorem 4. Let $G$ be a graph with $n$ vertices. Then,

$$
\varsigma_{(\alpha)}(G) \geq \sqrt{2 n Z-\frac{n^{2}}{4}\left(\delta_{(1)}^{\alpha}(G)-\delta_{(n)}^{\alpha}(G)\right)^{2}},
$$

where $\delta_{(1)}^{\alpha}(G)$ and $\delta_{(n)}^{\alpha}(G)$ are the largest and the smallest of $\left|\eta_{(i)}^{\alpha}(G)\right|, \quad$ respectively, and $Z=(1-\alpha)^{2} S+\left(\alpha^{2} / 2\right)$ $\sum_{i=1}^{n}\left(\operatorname{Tr}\left(v_{i}\right)-(2 W(G) / n)\right)^{2}$.

Proof. Let $\delta_{(1)}^{\alpha}(G) \geq \delta_{(2)}^{\alpha}(G) \geq, \ldots, \geq \delta_{(n)}^{\alpha}(G)$ be a nonincreasing sequence of $\left|\eta_{(i)}^{\alpha}(G)\right|$. According to Lemma 4, let $a_{i}=\delta_{(i)}^{\alpha}(G)$ and $b_{i}=1$, where $i=1, \ldots, n$, we have

$$
\begin{aligned}
& \left(\sum_{i=1}^{n}\left(\delta_{(i)}^{\alpha}(G)\right)^{2}\right)\left(\sum_{i=1}^{n} 1\right)-\left(\sum_{i=1}^{n} \delta_{(i)}^{\alpha}(G) \cdot 1\right)^{2} \\
& \leq \frac{n^{2}}{4}\left(\delta_{(1)}^{\alpha}(G)-\delta_{(n)}^{\alpha}(G)\right)^{2} .
\end{aligned}
$$

By equation (7), we have

$$
2 n Z-\left(\varsigma_{(\alpha)}(G)\right)^{2} \leq \frac{n^{2}}{4}\left(\delta_{(1)}^{\alpha}(G)-\delta_{(n)}^{\alpha}(G)\right)^{2} .
$$

Then,

$$
\varsigma_{(\alpha)}(G) \geq \sqrt{2 n Z-\frac{n^{2}}{4}\left(\delta_{(1)}^{\alpha}(G)-\delta_{(n)}^{\alpha}(G)\right)^{2}} .
$$

Lemma 5 (see [30]). Let $x_{1}>x_{2} \geq \ldots \geq x_{n}>0$ be $n$ real numbers. Then, 


$$
\sum_{i=1}^{n}\left|x_{i}-M\right|<\frac{n}{2} x_{1}
$$

where $M=\left(\sum_{1=1}^{n} x_{i} / n\right)$.

It follows from the above Proposition the following result holds directly.

Proposition 3. For a graph $G$ with $n$ vertices, let $\eta_{(1)}^{\alpha}(G)$ be the largest eigenvalue of $D_{\alpha}(G)$. For $\alpha \in[(1 / 2), 1)$,

$$
\varsigma_{(\alpha)}(G) \leq \frac{n}{2} \eta_{(1)}^{\alpha}(G)
$$

\section{Bounds for the $\alpha$-Distance Estrada Index of Graphs}

In this section, some bounds for $\alpha$-distance Estrada index are obtained in terms of Wiener index, the transmission of the vertex, spectral radius of $D_{\alpha}(G)$, and the vertex number. Furthermore, we give the relation between $\alpha$-distance Estrada index and $\alpha$-distance energy.

Next, we establish some bounds on the $\alpha$-distance Estrada index.

Lemma 6 (see [31]). Let $x_{1}, x_{2}, \ldots, x_{n}$ be nonnegative real numbers. Then, for $k \geq 2$,

$$
\sum_{i=1}^{n} x_{i}^{k} \leq\left(\sum_{i=1}^{n} x_{i}^{2}\right)^{(k / 2)}
$$

Theorem 5. Let $G$ be a graph with $n$ vertices. Then,

$$
\operatorname{DEE}_{(\alpha)}(G) \leq n+2 \alpha W(G)-1-\omega+e^{\omega},
$$

where $\omega=\sqrt{\alpha^{2} \sum_{i=1}^{n} \operatorname{Tr}^{2}\left(v_{i}\right)+2(1-\alpha)^{2} S}$.

Proof. Let $\sigma_{(1)}^{\alpha}(G), \ldots, \sigma_{(n)}^{\alpha}(G)$ be the eigenvalues of $D_{\alpha}(G)$. From equation (5) and Lemma 6, we have

$$
\begin{aligned}
\operatorname{DEE}_{(\alpha)}(G) & =n+2 \alpha W(G)+\sum_{i=1}^{n} \sum_{k=2}^{\infty} \frac{\left(\sigma_{(i)}^{\alpha}(G)\right)^{k}}{k !} \\
& \leq n+2 \alpha W(G)+\sum_{i=1}^{n} \sum_{k=2}^{\infty} \frac{\left|\sigma_{(i)}^{\alpha}(G)\right|^{k}}{k !} \\
& =n+2 \alpha W(G)+\sum_{k=2}^{\infty} \frac{1}{k !} \sum_{i=1}^{n}\left|\sigma_{(i)}^{\alpha}(G)\right|^{k} \\
& \leq n+2 \alpha W(G)+\sum_{k=2}^{\infty} \frac{1}{k !}\left(\sum_{i=1}^{n}\left(\sigma_{(i)}^{\alpha}(G)\right)^{2}\right)^{(k / 2)} \\
& =n+2 \alpha W(G)+\sum_{k=2}^{\infty} \frac{1}{k !}\left(\alpha^{2} \sum_{i=1}^{n} T r^{2}\left(v_{i}\right)+2(1-\alpha)^{2} S\right)^{(k / 2)} \\
& =n+2 \alpha W(G)-1-\omega+\sum_{k=0}^{\infty} \frac{1}{k !} \omega^{k} \\
& =n+2 \alpha W(G)-1-\omega+e^{\omega},
\end{aligned}
$$

where $\omega=\sqrt{\alpha^{2} \sum_{i=1}^{n} \operatorname{Tr}^{2}\left(v_{i}\right)+2(1-\alpha)^{2} S}$.

In the following, we obtained a lower bound on the $\alpha$-distance Estrada index by arithmetic-geometric inequality.

Theorem 6. Let $G$ be a graph with $n$ vertices. Then,

$$
\operatorname{DEE}_{(\alpha)}(G) \geq \sqrt{n+4 \alpha W(G)+n(n-1) e^{(4 \alpha W(G) / n)}} .
$$

Proof. Let $\sigma_{(1)}^{\alpha}(G), \ldots, \sigma_{(n)}^{\alpha}(G)$ be the eigenvalues of $D_{\alpha}(G)$. Then,

$$
\left(\operatorname{DEE}_{(\alpha)}(G)\right)^{2}=\sum_{i=1}^{n} e^{2 \sigma_{(i)}^{\alpha}(G)}+2 \sum_{1 \leq i<j \leq n} e^{\sigma_{(i)}^{\alpha}(G)} e^{\sigma_{(j)}^{\alpha}(G)} .
$$

From arithmetic-geometric inequality and equation (5), we obtain

$$
\begin{aligned}
2 \sum_{1 \leq i<j \leq n} e^{\sigma_{(i)}^{\alpha}(G)} e^{\sigma_{(j)}^{\alpha}(G)} & \geq n(n-1)\left(\prod_{1 \leq i<j \leq n} e^{\sigma_{(i)}^{\alpha}(G)} e^{\sigma_{(j)}^{\alpha}(G)}\right)^{(2 / n(n-1))} \\
& =n(n-1)\left(\left(\prod_{i=1}^{n} e^{\sigma_{(i)}^{\alpha}(G)}\right)^{n-1}\right)^{(2 / n(n-1))} \\
& =n(n-1)\left(e^{\sum_{i=1}^{n} \sigma_{(i)}^{\alpha}(G)}\right)^{(2 / n)} \\
& =n(n-1) e^{(4 \alpha W(G) / n)} .
\end{aligned}
$$

By means of a power-series expansion, we have

$$
\begin{aligned}
\sum_{i=1}^{n} e^{2 \sigma_{(i)}^{\alpha}(G)} & =\sum_{i=1}^{n} \sum_{k=0}^{\infty} \frac{\left(2 \sigma_{(i)}^{\alpha}(G)\right)^{k}}{k !} \\
& =n+4 \alpha W(G)+\sum_{i=1}^{n} \sum_{k=2}^{\infty} \frac{\left(2 \sigma_{(i)}^{\alpha}(G)\right)^{k}}{k !} \\
& \geq n+4 \alpha W(G) .
\end{aligned}
$$

By substituting equations (47) and (48) in equation (46), we see that

$$
\operatorname{DEE}_{(\alpha)}(G) \geq \sqrt{n+4 \alpha W(G)+n(n-1) e^{(4 \alpha W(G) / n)}} .
$$

Theorem 7. Let $G$ be a graph with $n$ vertices. Then,

$$
\operatorname{DEE}_{(\alpha)}(G) \geq e^{(2 W(G) / n)}+(n-1)+2 \alpha W(G)-\frac{2 W(G)}{n} \text {. }
$$

Proof. Let $f(x)=(x-1)-\ln x$, where $x>0$. Obviously, $f(x)$ is a decreasing function when $x \in(0,1]$, and $f(x)$ is increasing when $x \in[1,+\infty)$. Then, $f(x) \geq f(1)=0$, that is,

$$
x \geq 1+\ln x, \quad x>0,
$$

and the equality holds if and only if $x=1$. So, by this function and equation (5), we have 


$$
\begin{aligned}
\operatorname{DEE}_{(\alpha)}(G) & \geq e^{\sigma_{(1)}^{\alpha}(G)}+(n-1)+\sum_{k=2}^{n} \ln e^{\sigma_{(k)}^{\alpha}(G)} \\
& =e^{\sigma_{(1)}^{\alpha}(G)}+(n-1)+\sum_{k=2}^{n} \sigma_{(k)}^{\alpha}(G) \\
& =e^{\sigma_{(1)}^{\alpha}(G)}+(n-1)+2 \alpha W(G)-\sigma_{(1)}^{\alpha}(G),
\end{aligned}
$$

where $\sigma_{(1)}^{\alpha}(G), \ldots, \sigma_{(n)}^{\alpha}(G)$ are the eigenvalues of $D_{\alpha}(G)$.

Let $\Gamma(x)=e^{x}+(n-1)+2 \alpha W(G)-x$, where $x>0$. Clearly, $\Gamma(x)$ is an increasing function when $x \in(0,+\infty)$. From Lemma 1, we have

$$
\sigma_{(1)}^{\alpha}(G) \geq \frac{2 W(G)}{n} \geq 0
$$

Then,

$$
\Gamma\left(\sigma_{(1)}^{\alpha}(G)\right) \geq \Gamma\left(\frac{2 W(G)}{n}\right)
$$

Hence,

$$
\operatorname{DEE}_{(\alpha)}(G) \geq e^{(2 W(G) / n)}+(n-1)+2 \alpha W(G)-\frac{2 W(G)}{n} .
$$

From Theorem 7, we have the following result.

Corollary 2. Let $G$ be a transmission regular graph with $n$ vertices. Let $\operatorname{Tr}(u)=r$ for each $u \in V(G)$. Then,

$$
\operatorname{DEE}_{(\alpha)}(G) \geq e^{r}+(n-1)+\alpha n r-r .
$$

We are inspired by literature [32], and we give Theorems 8 and 9 as follows.

Lemma 7 (see [33]). For $a_{1}, a_{2}, \ldots, a_{n} \geq 0$ and $p_{1}, p_{2}, \ldots, p_{n} \geq 0$ such that $\sum_{i=1}^{n} p_{i}=1$. Then,

$$
\sum_{i=1}^{n} p_{i} a_{i}-\prod_{i=1}^{n} a_{i}^{p_{i}} \geq n T\left(\frac{1}{n} \sum_{i=1}^{n} a_{i}-\prod_{i=1}^{n} a_{i}^{(1 / n)}\right),
$$

where $T=\min \left\{p_{1}, p_{2}, \ldots, p_{n}\right\}$. Equality holds if and only if $a_{1}=a_{2}=, \ldots,=a_{n}$.

Theorem 8. Let $G$ be a graph with $n$ vertices. Then,

$$
\operatorname{DEE}_{(\alpha)}(G) \geq e^{\sigma_{(1)}^{\alpha}(G)}+2(n-1) \Delta-(n-1) e^{(2 \alpha W(G) / n)},
$$

where $\Delta=e^{\left(2(n-1) \alpha W(G)+n\left(\sigma_{(2)}^{\alpha}(G)+, \ldots,+\sigma_{(n)}^{\alpha}(G)\right) / 2 n(n-1)\right)}$. Equality holds if and only if $G \cong n K_{1}$.

Proof. Let $p_{1}=(1 / 2 n), \quad p_{i}=(2 n-1 / 2 n(n-1))$ for $i=2, \ldots, n, \quad a_{i}=e^{\sigma_{(i)}^{\alpha}(G)}$ for $i=2, \ldots, n$. Obviously, $T=\min \{(1 / 2 n),(2 n-1 / 2 n(n-1))\}=(1 / 2 n)$, and according to Lemma 7 , we have

$$
\begin{aligned}
& \frac{e^{\sigma_{(1)}^{\alpha}(G)}}{2 n}+\frac{2 n-1}{2 n(n-1)} \sum_{i=2}^{n} e^{\sigma_{(i)}^{\alpha}(G)}-\Delta \\
& \geq \frac{1}{2}\left(\frac{1}{n} \sum_{i=1}^{n} e^{\sigma_{(i)}^{\alpha}(G)}-\prod_{i=1}^{n} e^{\sigma_{(i)}^{\alpha}(G) / n}\right),
\end{aligned}
$$

where $\Delta=e^{\left(\sigma_{(1)}^{\alpha}(G) / 2 n\right)} \prod_{i=2}^{n} e^{\left((2 n-1) \sigma_{(i)}^{\alpha}(G) / 2 n(n-1)\right)}$.

By equation (5), we have

$$
\begin{aligned}
\Delta= & e^{\left(\sigma_{(1)}^{\alpha}(G) / 2 n\right)} \prod_{i=2}^{n} e^{\left((2 n-1) \sigma_{(i)}^{\alpha}(G) / 2 n(n-1)\right)} \\
= & e^{\left((n-1)\left(2 \alpha W(G)-\sum_{i=2}^{n} \sigma_{(i)}^{\alpha}(G)\right) / 2 n(n-1)\right)} \\
& \cdot e^{\left((2 n-1) \sum_{i=2}^{n} \sigma_{(i)}^{\alpha}(G) / 2 n(n-1)\right)} \\
= & e^{\left(2(n-1) \alpha W(G)+n\left(\sigma_{(2)}^{\alpha}(G)+, \ldots,+\sigma_{(n)}^{\alpha}(G)\right) / 2 n(n-1)\right)} .
\end{aligned}
$$

Since

$$
\begin{aligned}
& \frac{e^{\sigma_{(1)}^{\alpha}(G)}}{2 n}+\frac{2 n-1}{2 n(n-1)}\left(\sum_{i=1}^{n} e^{\sigma_{(i)}^{\alpha}(G)-e^{\sigma_{(1)}^{\alpha}(G)}}\right)-\Delta \\
& \geq \frac{1}{2 n} \sum_{i=1}^{n} e^{\sigma_{(i)}^{\alpha}(G)}-\frac{1}{2} e^{(2 \alpha W(G) / n)},
\end{aligned}
$$

then

$$
\begin{aligned}
\operatorname{DEE}_{(\alpha)}(G)= & \sum_{i=1}^{n} e^{\sigma_{(i)}^{\alpha}(G)} \geq e^{\sigma_{(1)}^{\alpha}(G)}+2(n-1) \Delta \\
& -(n-1) e^{(2 \alpha W(G) / n)} .
\end{aligned}
$$

Equality holds, that is, $e^{\sigma_{(1)}^{\alpha}(G)}=e^{\sigma_{(2)}^{\alpha}(G)}=, \ldots,=e^{\sigma_{(n)}^{\alpha}(G)}$ if and only if $G \cong n K_{1}$.

Lemma 8 (see [34]). For $a_{1}, a_{2}, \ldots, a_{n} \geq 0$. Then,

$$
\begin{aligned}
& n\left(\frac{1}{n} \sum_{i=1}^{n} a_{i}-\left(\prod_{i=1}^{n} a_{i}\right)^{(1 / n)}\right) \leq \Psi \\
& \leq n(n-1)\left(\frac{\sum_{i=1}^{n} a_{i}}{n}-\left(\prod_{i=1}^{n} a_{i}\right)^{(1 / n)}\right),
\end{aligned}
$$

where $\Psi=n \sum_{i=1}^{n} a_{i}-\left(\sum_{i=1}^{n} \sqrt{a_{i}}\right)^{2}$.

Theorem 9. Let $G$ be a graph with $n$ vertices. Then,

$$
\begin{gathered}
\frac{\left(\sum_{i=1}^{n} e^{\left(\sigma_{(i)}^{\alpha}(G) / 2\right)}\right)^{2}-n e^{(2 \alpha W(G) / n)}}{n-1} \leq \operatorname{DEE}_{(\alpha)}(G) \\
\leq\left(\sum_{i=1}^{n} e^{\left(\sigma_{(i)}^{\alpha}(G) / 2\right)}\right)-n(n-1) e^{(2 \alpha W(G) / n)}
\end{gathered}
$$


Proof. Let $a_{i}=e^{\sigma_{(i)}^{\alpha}(G)}$ for $i=1,2, \ldots, n$, by Lemma 8 , we have

$$
\begin{aligned}
& \sum_{i=1}^{n} e^{\sigma_{(i)}^{\alpha}(G)}-n\left(\prod_{i=1}^{n} e^{\sigma_{(i)}^{\alpha}(G)}\right)^{(1 / n)} \leq \Psi \\
& \quad \leq n(n-1)\left(\frac{\sum_{i=1}^{n} e^{\sigma_{(i)}^{\alpha}(G)}}{n}-\left(\prod_{i=1}^{n} e^{\sigma_{(i)}^{\alpha}(G)}\right)^{(1 / n)}\right)
\end{aligned}
$$

where $\Psi=n \sum_{i=1}^{n} e^{\sigma_{(i)}^{\alpha}(G)}-\left(\sum_{i=1}^{n} e^{\left(\sigma_{(i)}^{\alpha}(G) / 2\right)}\right)^{2}$.

Analyzing the left and right side of the previous inequality, respectively, by equation (5), we have

$$
\begin{gathered}
\sum_{i=1}^{n} e^{\sigma_{(i)}^{\alpha}(G)}-n\left(\prod_{i=1}^{n} e^{\sigma_{(i)}^{\alpha}(G)}\right)^{(1 / n)} \leq n \sum_{i=1}^{n} e^{\sigma_{(i)}^{\alpha}(G)}-\left(\sum_{i=1}^{n} e^{\left(\sigma_{(i)}^{\alpha}(G) / 2\right)}\right)^{2} \\
(n-1) \sum_{i=1}^{n} e^{\sigma_{(i)}^{\alpha}(G)} \geq\left(\sum_{i=1}^{n} e^{\left.\left(\sigma_{(i)}^{\alpha}(G) / 2\right)\right)^{2}-n e^{(2 \alpha W(G) / n)}}\right. \\
\operatorname{DEE}_{(\alpha)}(G) \geq \frac{\left(\sum_{i=1}^{n} e^{\left(\sigma_{(i)}^{\alpha}(G) / 2\right)}\right)^{2}-n e^{(2 \alpha W(G) / n)}}{n-1}, \\
n \sum_{i=1}^{n} e^{\sigma_{(i)}^{\alpha}(G)}-\left(\sum_{i=1}^{n} e^{\left(\sigma_{(i)}^{\alpha}(G) / 2\right)}\right)^{2} \\
\operatorname{DEE}_{(\alpha)}(G) \leq\left(\sum_{i=1}^{n} e^{\left(\sigma_{(i)}^{\alpha}(G) / 2\right)}\right)-n(n-1) e^{(2 \alpha W(G) / n)} . \\
n
\end{gathered}
$$

Also, the relation between $\alpha$-distance Estrada index and

$$
\operatorname{DEE}_{(\alpha)}(G) \leq e^{(2 \alpha W(G) / n)}\left(n-1-\varsigma_{(\alpha)}(G)+e^{\zeta_{(\alpha)}(G)}\right) .
$$
$\alpha$-distance energy are established.

Theorem 10. Let $G$ be a graph with $n$ vertices. Then,

Proof. By the definition of $\alpha$-distance energy, we have

$$
\begin{aligned}
\operatorname{DEE}_{(\alpha)}(G) & =e^{(2 \alpha W(G) / n)}\left(\sum_{i=1}^{n} e^{\sigma_{(i)}^{\alpha}(G)-(2 \alpha W(G) / n)}\right) \\
& =e^{(2 \alpha W(G) / n)}\left(n+\sum_{i=1}^{n} \sum_{k=2}^{\infty} \frac{\left(\sigma_{(i)}^{\alpha}(G)-(2 \alpha W(G) / n)\right)^{k}}{k !}\right) \\
& \leq e^{(2 \alpha W(G) / n)}\left(n+\sum_{i=1}^{n} \sum_{k=2}^{\infty} \frac{\left|\sigma_{(i)}^{\alpha}(G)-(2 \alpha W(G) / n)\right|^{k}}{k !}\right) \\
& \leq e^{(2 \alpha W(G) / n)}\left(n+\sum_{k=2}^{\infty} \frac{1}{k !}\left(\varsigma_{(\alpha)}(G)\right)^{k}\right) \\
& =e^{(2 \alpha W(G) / n)}\left(n-1-\varsigma_{(\alpha)}(G)+e^{\varsigma_{(\alpha)}(G)}\right) .
\end{aligned}
$$




\section{Conclusion}

It is well-known that the graph energy and the Estrada index of graphs are important topics in graph theory. The $\alpha$-distance matrix is an extension of the distance matrix. And the $\alpha$-distance energy and $\alpha$-distance Estrada index are generalized distance energy and distance Estrada index of graphs, respectively. In this paper, we establish some bounds on $\alpha$-distance energy and $\alpha$-distance Estrada index of $G$. Furthermore, a new lower bound for the $\alpha$-distance Estrada index in relation to the $\alpha$-distance energy of the graph $G$ is given.

\section{Data Availability}

No data were used to support this study.

\section{Conflicts of Interest}

The authors declare to have no conflicts of interest.

\section{Authors' Contributions}

All authors contributed equally to the writing of this paper. All authors read and approved the final manuscript.

\section{Acknowledgments}

This work was supported by the National Natural Science Foundation of China (nos. 11801115 and 11601102), the Natural Science Foundation of the Heilongjiang Province (no. QC2018002), and the Fundamental Research Funds for the Central Universities.

\section{References}

[1] R. L. Graham and L. Lovász, "Distance matrix polynomials of trees," Advances in Mathematics, vol. 29, no. 1, pp. 60-88, 1978.

[2] R. L. Graham and H. O. Pollak, "On the addressing problem for loop switching," Bell System Technical Journal, vol. 50, no. 8, pp. 2495-2519, 1971.

[3] P. M. Winkler, "Isometric embedding in products of complete graphs," Discrete Applied Mathematics, vol. 7, no. 2, pp. 221-225, 1984.

[4] M. Aouchiche and P. Hansen, "Two Laplacians for the distance matrix of a graph," Linear Algebra and its Applications, vol. 439, no. 1, pp. 21-33, 2013.

[5] V. Nikiforov, "Merging the A- and Q-spectral theories," Applicable Analysis and Discrete Mathematics, vol. 11, no. 1, pp. 81-107, 2017.

[6] S.-Y. Cui, J.-X. He, and G.-X. Tian, "The generalized distance matrix," Linear Algebra and its Applications, vol. 563, pp. 1-23, 2019.

[7] H. Lin and B. Zhou, "The effect of graft transformations on distance signless Laplacian spectral radius," Linear Algebra and its Applications, vol. 504, pp. 433-461, 2016.

[8] R. Xing, B. Zhou, and J. Li, "On the distance signless Laplacian spectral radius of graphs," Linear and Multilinear Algebra, vol. 62, no. 10, pp. 1377-1387, 2014.
[9] R. Xing and B. Zhou, "On the distance and distance signless Laplacian spectral radii of bicyclic graphs," Linear Algebra and its Applications, vol. 439, no. 12, pp. 3955-3963, 2013.

[10] S. Y. Cui, G. X. Tian, and L. Zheng, "On the generalized distance spectral radius of graphs," 2019, http://arxiv.org/abs/ 1901.07695.

[11] H. Y. Guo and B. Zhou, "On the distance $\alpha$-spectral radius of a connected graph,” 2019, http://arxiv.org/abs/1901.10180.

[12] H. Lin, J. Xue, and J. Shu, "On the $D_{\alpha}$-spectra of graphs," Linear and Multilinear Algebra, pp. 1-23.

[13] K. C. Das, M. Aouchiche, and P. Hansen, "On (distance) Laplacian energy and (distance) signless Laplacian energy of graphs," Discrete Applied Mathematics, vol. 243, pp. 172-185, 2018.

[14] I. Gutman, "Acyclic systems with extremal Hückel $\pi$-electron energy," Theoretica Chimica Acta, vol. 45, no. 2, pp. 79-87, 1977.

[15] I. Gutman, "The energy of a graph," Ber. Math-Statist. Sekt. Forschungsz. Graz., vol. 103, pp. 1-22, 1978.

[16] G. Indulal and I. Gutman, "On the distance spectra of some graphs," Mathematical Communications, vol. 13, no. 1, pp. 123-131, 2008.

[17] B. J. McClelland, "Properties of the latent roots of a matrix: the estimation of $\pi$-electron energies," The Journal of Chemical Physics, vol. 54, no. 2, pp. 640-643, 1971.

[18] J. H. Koolen and V. Moulton, "Maximal energy graphs," Advances in Applied Mathematics, vol. 26, no. 1, pp. 47-52, 2001.

[19] R. A. Brualdi, Energy of a Graph, Notes for AIM Workshop on Spectra of Families of Matrices Described by Graphs, Digraphs and Sign Patterns, http://aimath.org/pastworkshops/ matrixspectrumrep.pdf/, 2006.

[20] E. Estrada, "Characterization of 3D molecular structure," Chemical Physics Letters, vol. 319, no. 5-6, pp. 713-718, 2000.

[21] B. Zhou and I. Gutman, "More on the laplacian Estrada index," Applicable Analysis and Discrete Mathematics, vol. 3, no. 2, pp. 371-378, 2009.

[22] E. Estrada, The Structure of Complex Networks: Theory and Applications, Oxford University Press, Oxford, UK, 2012.

[23] E. Estrada and J. A. Rodríguez-Velázquez, "Subgraph centrality in complex networks," Physical Review E, vol. 71, p. 581, 2005.

[24] E. Estrada and J. A. Rodríguez-Velázquez, "Spectral measures of bipartivity in complex networks," Physical Review E, vol. 72, p. 046, 2005.

[25] Y. Shang, "Biased edge failure in scale-free networks based on natural connectivity," Indian Journal of Physics, vol. 86, no. 6, pp. $485-488,2012$.

[26] A. D. Glźngör and S. B. Bozkurt, "On the distance Estrada index of graphs," Hacettepe Journal of Mathematics and Statistics, vol. 38, no. 3, pp. 277-283, 2009.

[27] H. Dong and X. Guo, "Ordering trees by their wiener indices," MATCH Communications in Mathematical and in Computer Chemistry, vol. 25, pp. 527-540, 2006.

[28] N. Ozeki, "On the estimation of the inequality by the maximum," Journal of the College of Arts and Sciences, Chiba University, vol. 5, pp. 199-203, 1968.

[29] G. Polya and G. Szego, Problems and Theorems in Analysis. Series, Integral Calculus. Theory of Functions, Springer, Berlin, Germany, 1972.

[30] R. C. Díłaz and O. Rojo, "Sharp upper bounds on the distance energies of a graph," Linear Algebra Appl, vol. 54, pp. 55-75, 2018. 
[31] N. J. Rad, A. Jahanbani, and I. Gutman, "Zagreb energy and Zagreb Estrada index of graphs," MATCH Communications in Mathematical and in Computer Chemistry, vol. 79, pp. 371386, 2018.

[32] R. Jonnathan, "A note on new bounds for the Estrada Index," Linear Algebra and its Applications, vol. 580, pp. 121-127, 2019.

[33] J. Li, J.-M. Guo, W. C. Shiu, Ş. B. B. Altındağ, and D. Bozkurt, "Bounding the sum of powers of normalized Laplacian eigenvalues of a graph," Applied Mathematics and Computation, vol. 324, pp. 82-92, 2018.

[34] P. B. Sarasija and R. Binthiya, "Bounds on the Seidel energy of strongly quotient graphs," Journal of Chemical and Pharmaceutical Sciences, vol. 10, 2007. 